\title{
A POROSITY RESULT IN CONVEX MINIMIZATION
}

\author{
P. G. HOWLETT AND A. J. ZASLAVSKI
}

Received 1 August 2003

We study the minimization problem $f(x) \rightarrow \min , x \in C$, where $f$ belongs to a complete metric space $M$ of convex functions and the set $C$ is a countable intersection of a decreasing sequence of closed convex sets $C_{i}$ in a reflexive Banach space. Let $\mathscr{F}_{F}$ be the set of all $f \in \mathcal{M}$ for which the solutions of the minimization problem over the set $C_{i}$ converge strongly as $i \rightarrow \infty$ to the solution over the set $C$. In our recent work we show that the set $\mathscr{F}$ contains an everywhere dense $G_{\delta}$ subset of $\mathcal{M}$. In this paper, we show that the complement $\mathcal{M} \backslash \mathscr{F}$ is not only of the first Baire category but also a $\sigma$-porous set.

\section{Introduction}

Let $X$ be a reflexive Banach space with the norm $\|\cdot\|$ and let

$$
C_{\infty}=\bigcap_{i=1}^{\infty} C_{i} \neq \varnothing,
$$

where $C_{i+1} \subset C_{i}$ for each $i=1,2, \ldots$ and where each $C_{i}$ is a closed convex subset of $X$. We study the minimization problem

$$
f(x) \longrightarrow \min , \quad x \in C_{\infty}
$$

where $f$ belongs to a complete metric space $\mathcal{M}$ of convex functions defined on $C_{1}$. In [8] we show that for a generic function $f \in \mathcal{M}$ the solutions of the minimization problem over the set $C_{i}$ converge strongly as $i \rightarrow \infty$ to the solution over the set $C_{\infty}$.

When we say that a certain property holds for a generic element of a complete metric space $Y$ we mean that the set of points which have this property contains a $G_{\delta}$ everywhere dense subset of $Y$. A set $G$ is said to belong to the class $G_{\delta}$ if it can be expressed as a countable intersection of open sets. Such an approach, when a certain property is investigated for the whole space $Y$, and not just for a single point in $Y$, has already been successfully applied in many areas of analysis. We mention, for instance, the theory of dynamical systems $[5,13,15]$, optimization $[3,7,8,9,11,12]$, variational analysis [1], approximation theory [6], the calculus of variations $[2,4,9,18]$ and optimal control $[19,20,21]$. 
In this paper, we study the set of all functions $f \in \mathcal{M}$ for which the solutions of the minimization problem over the sets $C_{i}$ converge strongly as $i \rightarrow \infty$ to the solution of the minimization problem over the set $C_{\infty}$. We show that the complement of this set is not only of the first Baire category but also a $\sigma$-porous set.

We now recall the concept of porosity $[5,6,7,12]$.

Let $(Y, d)$ be a complete metric space. We denote by $B_{d}(y, r)$ the closed ball of center $y \in Y$ and radius $r>0$. A subset $E \subset Y$ is called porous in $(Y, d)$ if there exist $\alpha \in(0,1]$ and $r_{0}>0$ such that for each $r \in\left(0, r_{0}\right]$ and each $y \in Y$ there exists $z \in Y$ for which

$$
B_{d}(z, \alpha r) \subset B_{d}(y, r) \backslash E .
$$

Hence every ball under a certain size includes a smaller ball of fixed proportional size that is contained in the complement of $E$.

Remark 1.1. In the above definition of porosity it is known that the point $y$ can be assumed to belong to $E$.

A subset of the space $Y$ is called $\sigma$-porous in $(Y, d)$ if it is a countable union of porous subsets in $(Y, d)$.

Other notions of porosity have been used in the literature $[16,17]$. We use the rather strong notion which appears in $[5,6,7,12]$.

Since porous sets are nowhere dense, all $\sigma$-porous sets are of the first category. That is, each $\sigma$-porous set can be expressed as a countable union of nowhere dense subsets. If $Y$ is a finite-dimensional Euclidean space, then all $\sigma$-porous sets are of Lebesgue measure zero. In fact, the class of $\sigma$-porous sets in such a space is smaller than the class of sets which have measure zero and are of the first category [16]. Furthermore every complete metric space without isolated points contains a closed nowhere dense set which is not $\sigma$-porous [17].

To differentiate between porous and nowhere dense sets note that if $E \subset Y$ is nowhere dense, $y \in Y$, and $r>0$, then there is a point $z \in Y$ and a number $s>0$ such that $B_{d}(z, s) \subset$ $B_{d}(y, r) \backslash E$. If, however, $E$ is also porous, then for small enough $r$ we can choose $s=\alpha r$, where $\alpha \in(0,1)$ is a constant which depends only on $E$.

\section{The main result}

We use the convention that $\infty-\infty=0$ and $\infty / \infty=1$. Let $X$ be a reflexive Banach space with the norm $\|\cdot\|$ and let

$$
C_{\infty}=\bigcap_{i=1}^{\infty} C_{i} \neq \varnothing,
$$

where $C_{i+1} \subset C_{i}$ for each $i=1,2, \ldots$ and where each $C_{i}$ is a closed convex subset of $X$. Let $\varphi: C_{1} \rightarrow R^{1}$ satisfy

$$
\varphi(x) \longrightarrow \infty \text { as }\|x\| \longrightarrow \infty
$$


Denote by $\mathcal{M}$ the set of all convex lower semicontinuous functions $f: C_{1} \rightarrow R^{1} \cup\{\infty\}$ which are not identically infinity on $C_{\infty}$ and satisfy

$$
f(x) \geq \varphi(x) \quad \forall x \in C_{1} .
$$

For each $f \in M$ and each nonempty set $C \subset C_{1}$ set

$$
\inf (f ; C)=\inf \{f(x) \mid x \in C\} .
$$

It is well known that for each $f \in \mathcal{M}$ and each $i \in\{1,2, \ldots\} \cup\{\infty\}$, the following minimization problem:

$$
\left(P_{i}^{f}\right) f(x) \rightarrow \min , x \in C_{i}
$$

has a solution. Denote by $M_{1}$ the set of all finite-valued functions $f \in \mathcal{M}$ and by $M_{2}$ the set of all finite-valued continuous functions $f \in \mathcal{M}$. Next we endow the set $\mathcal{M}$ with a metric $d$. For each $f, g \in \mathcal{M}$ and each $m \in \mathbb{N}$, we first set

$$
d_{m}(f, g)=\sup \left\{|f(x)-g(x)|: x \in C_{1} \text { and }\|x\| \leq m\right\}
$$

and then define

$$
d(f, g)=\sum_{m=1}^{\infty} 2^{-m}\left[d_{m}(f, g)\left(d_{m}(f, g)+1\right)^{-1}\right]
$$

(see the convention at the start of this section). We adopt the convention that the supremum of the empty set is zero. Clearly $(M, d)$ is a complete metric space. It is also not difficult to see that the collection of sets

$$
E(m, \delta)=\left\{(f, g) \in M \times M|| f(x)-g(x) \mid \leq \delta \text { for each } x \in C_{1} \text { satisfying }\|x\| \leq m\right\},
$$

where $m \in \mathbb{N}$ and $\delta>0$, is a base for the uniformity generated by the metric $d$. Evidently $M_{1}$ and $M_{2}$ are closed subsets of the metric space $(M, d)$. In the sequel we assign to all these spaces the same metric $d$.

In [8] for a function $f \in M_{2}$ we studied the convergence of solutions to the problem $\left(P_{i}^{f}\right)$ for each $i=1,2, \ldots$ to a solution of the problem $\left(P_{\infty}^{f}\right)$. If $X$ is a Hilbert space with inner product $\langle\cdot, \cdot\rangle$ and

$$
f(x)=\langle x, x\rangle \quad \text { for } x \in X,
$$

then this convergence property was established by Semple [14]. A similar result was also obtained for certain Banach spaces and the distance function by Israel Jr. and Reich [10]. In [8] we showed that the convergence property holds for most functions $f \in M_{2}$. More precisely, we considered the metric space $\left(M_{2}, d\right)$ with

$$
\varphi(x)=a_{1}\|x\|-a_{2} \quad \text { for } x \in C_{1},
$$

where $a_{1}$ and $a_{2}$ are positive numbers, and showed that there exists a subset of $\mu_{2}$ which is a countable intersection of open everywhere dense sets such that for each function 
belonging to this subset the convergence property holds. Note that this result is true for reflexive Banach spaces but not necessarily for nonreflexive Banach spaces. For more information consider [8, Examples 1 and 2].

In this paper for the spaces $M, M_{1}$, and $M_{2}$ we show that the complements of subsets of functions which have the convergence property are not only of the first Baire category but are also $\sigma$-porous sets. We will establish the following result.

Theorem 2.1. Let $\mathscr{A}$ be either $M, M_{1}$, or $M_{2}$. There exists a set $\mathscr{F} \subset \mathscr{A}$ such that the complement $\mathscr{A} \backslash \mathscr{F}$ is $\sigma$-porous in $(\mathscr{A}, d)$ and such that for each $f \in \mathscr{F}$ the following properties hold.

$\left(\mathrm{P}_{1}\right)$ There exists a unique point $x_{f} \in C_{\infty}$ such that $f\left(x_{f}\right)=\inf \left(f ; C_{\infty}\right)$.

$\left(\mathrm{P}_{2}\right)$ For each $i=1,2, \ldots$, let $x_{i} \in C_{i}$ with $f\left(x_{i}\right)=\inf \left(f ; C_{i}\right)$. Then $\left\|x_{i}-x_{f}\right\| \rightarrow 0$ as $i \rightarrow \infty$.

$\left(\mathrm{P}_{3}\right)$ For each $\epsilon>0$ there exist a neighborhood $U$ of $f$ in $(\mathscr{A}, d)$, a number $\delta>0$, and a natural number $p$ such that for each $g \in U$, each $i \in\{p, p+1, \ldots\} \cup\{\infty\}$, and each $y \in C_{i}$ satisfying

$$
g(y) \leq \inf \left(g ; C_{i}\right)+\delta
$$

the relation $\left\|y-x_{f}\right\| \leq \epsilon$ also holds.

\section{Auxiliary results}

We begin with a lemma.

Lemma 3.1. Let $f \in \mathcal{M}$ and $\delta>0$. Choose $m=m(f) \in \mathbb{N}$ so that

$$
\left[z \in C_{1} \text { and } \varphi(z) \leq \inf \left(f ; C_{\infty}\right)+1\right] \Longrightarrow\|z\| \leq m
$$

and define $U(m, \delta)=\{g \in \mathcal{M} \mid(f, g) \in E(m, \delta)\}$. Then for each $i \in\{1,2, \ldots\} \cup\{\infty\}$ and each $g \in U(m, \delta)$,

$$
\inf \left(g ; C_{i}\right) \leq \inf \left(f ; C_{i}\right)+\delta
$$

Proof of Lemma 3.1. Let $i \in\{1,2, \ldots\} \cup\{\infty\}$ and $z \in C_{i}$ with $f(z) \leq \inf \left(f ; C_{i}\right)+1$. Since $C_{i} \subset C_{1}$ and $\varphi(z) \leq f(z) \leq \inf \left(f, C_{i}\right)+1 \leq \inf \left(f, C_{\infty}\right)+1$ it follows that $\|z\| \leq m$ and hence that $|f(z)-g(z)| \leq \delta$. Therefore for each $i \in\{1,2, \ldots\} \cup\{\infty\}$,

$$
\begin{aligned}
\inf \left(g ; C_{i}\right) & \leq \inf \left\{g(z) \mid z \in C_{i} \text { and } f(z) \leq \inf \left(f ; C_{i}\right)+1\right\} \\
& \leq \inf \left\{f(z)+\delta \mid z \in C_{i} \text { and } f(z) \leq \inf \left(f ; C_{i}\right)+1\right\} \\
& \leq \inf \left\{f(z) \mid z \in C_{i} \text { and } f(z) \leq \inf \left(f ; C_{i}\right)+1\right\}+\delta \\
& \leq \inf \left(f ; C_{i}\right)+\delta .
\end{aligned}
$$

The next proposition introduces a useful property. 
Proposition 3.2. Let $f \in M$. Suppose there exists a unique point $x_{f} \in C_{\infty}$ such that $f\left(x_{f}\right)=\inf \left(f ; C_{\infty}\right)$ and suppose that the following property holds.

$\left(\mathrm{P}_{4}\right)$ For each $\epsilon>0$ there exist $\delta=\delta(\epsilon)>0$ and $p=p(\epsilon) \in \mathbb{N}$ such that for each $i \in$ $\{p, p+1, \ldots\} \cup\{\infty\}$ and each $y \in C_{i}$ satisfying $f(y) \leq \inf \left(f ; C_{i}\right)+\delta$ the inequality $\left\|y-x_{f}\right\| \leq \epsilon$ is valid.

Then properties $\left(P_{1}\right),\left(P_{2}\right)$, and $\left(P_{3}\right)$ also hold.

Proof of Proposition 3.2. Property $\left(\mathrm{P}_{1}\right)$ holds by virtue of the first assumption. For each $i=1,2, \ldots$, we choose $x_{i} \in C_{i}$ with $f\left(x_{i}\right)=\inf \left(f ; C_{i}\right)$. The truth of property $\left(\mathrm{P}_{4}\right)$ clearly implies the truth of $\left(\mathrm{P}_{2}\right)$. We will show that property $\left(\mathrm{P}_{3}\right)$ also holds. Choose $\epsilon>0$. By property $\left(\mathrm{P}_{4}\right)$ there exist $\delta \in(0,1 / 2)$ and $p \in \mathbb{N}$ such that if

$$
i \in\{p, p+1, \ldots\} \cup\{\infty\}, \quad y \in C_{i}, \quad f(y) \leq \inf \left(f ; C_{i}\right)+3 \delta,
$$

then $\left\|y-x_{f}\right\| \leq \epsilon$. Choose $m=m(f) \in \mathbb{N}$ and define $U=U(m, \delta)$ as in Lemma 3.1. Let $i \in\{p, p+1, \ldots\} \cup\{\infty\}$ and $g \in U$. Assume that $z \in C_{i}$ with $g(z) \leq \inf \left(g ; C_{i}\right)+\delta$. From Lemma 3.1 it follows that

$$
\varphi(z) \leq g(z) \leq \inf \left(g ; C_{i}\right)+\delta \leq \inf \left(f ; C_{i}\right)+2 \delta<\inf \left(f ; C_{\infty}\right)+1
$$

and hence $\|z\| \leq m$ and $|f(z)-g(z)| \leq \delta$. Since

$$
f(z) \leq g(z)+\delta \leq \inf \left(g ; C_{i}\right)+2 \delta \leq \inf \left(f ; C_{i}\right)+3 \delta
$$

it follows that $\left\|z-x_{f}\right\| \leq \epsilon$. Hence property $\left(\mathrm{P}_{3}\right)$ is also true.

Lemma 3.3. Let $f \in \mathcal{M}$. Then

$$
\liminf _{i \rightarrow \infty}\left(f ; C_{i}\right)=\inf \left(f ; C_{\infty}\right) .
$$

Lemma 3.3 is similar to [8, Lemma 2.1]. The proof is also similar and is omitted.

\section{Proof of the main result}

It is convenient to split the proof into several smaller parts. We use the notation $\mathscr{A}$ to denote either $M, M_{1}$, or $M_{2}$.

Lemma 4.1. For each $n \in \mathbb{N}$ let $\mathscr{F}_{n}$ denote the set of all $f \in \mathscr{A}$ with the following property.

$\left(Q_{1}\right)$ There exist $x_{n} \in C_{\infty}, \delta_{n}>0$, and $p_{n} \in \mathbb{N}$ such that for $i \in\left\{p_{n}, p_{n}+1, \ldots\right\} \cup\{\infty\}$ and each $y \in C_{i}$ with $f(y) \leq \inf \left(f ; C_{i}\right)+\delta_{n}$ the inequality $\left\|y-x_{n}\right\| \leq 1 / n$ is valid. If $f \in \mathscr{F}=\bigcap_{n=1}^{\infty} \mathscr{F}_{n}$, then properties $\left(P_{1}\right),\left(P_{2}\right)$, and $\left(P_{3}\right)$ all hold.

Proof of Lemma 4.1. Let $x_{f} \in C_{\infty}$ satisfy $f\left(x_{f}\right)=\inf \left(f ; C_{\infty}\right)$. By property $\left(Q_{1}\right)$ with $i=\infty$ and $y=x_{f}$ we know that

$$
\| x_{f}-x_{n}|| \leq \frac{1}{n}
$$


for all $n \in \mathbb{N}$. Hence $x_{f}=\lim _{n \rightarrow \infty} x_{n}$. Therefore $x_{f}$ is the unique minimizer of $f$ on $C_{\infty}$. Let $\epsilon>0$ and $n \in \mathbb{N}$ be such that $n>2 / \epsilon$. For each $i \in\left\{p_{n}, p_{n}+1, \ldots\right\} \cup\{\infty\}$ and each $y \in C_{i}$ with $f(y) \leq \inf \left(f ; C_{i}\right)+\delta_{n}$, it follows from property $\left(Q_{1}\right)$ that

$$
\left\|y-x_{n}\right\| \leq \frac{1}{n}
$$

Hence $\left\|y-x_{f}\right\| \leq \epsilon$. Thus property $\left(\mathrm{P}_{4}\right)$ is valid and hence also properties $\left(\mathrm{P}_{1}\right),\left(\mathrm{P}_{2}\right)$, and $\left(\mathrm{P}_{3}\right)$.

Remark 4.2. To complete the proof of Theorem 2.1, we need to show that $\mathscr{A} \backslash \mathscr{F}$ is $\sigma$ porous in $(\mathscr{A}, d)$. Since $\mathscr{A} \backslash \mathscr{F}_{F}=\bigcup_{n=1}^{\infty}\left(\mathscr{A} \backslash \mathscr{F}_{n}\right)$ it is sufficient to show that the set $\mathscr{A} \backslash \mathscr{F}_{n}$ is $\sigma$-porous in $(\mathscr{A}, d)$ for any $n \in \mathbb{N}$. For each $m \in \mathbb{N}$ denote by $E_{m}$ the subset of all $f \in \mathscr{A}$ with the following property.

$\left(Q_{2}\right)$ If $x \in C_{1}$ and $\varphi(x) \leq \inf \left(f ; C_{\infty}\right)+1$, then $\|x\| \leq m$.

Since $\bigcup_{m=1}^{\infty} E_{m}=\mathscr{A}$ and $\mathscr{A} \backslash \mathscr{F}_{n}=\bigcup_{m=1}^{\infty}\left(E_{m} \backslash \mathscr{F}_{n}\right)$ it is sufficient to show that for each $m, n \in \mathbb{N}$ the set $E_{m} \backslash \mathscr{F}_{n}$ is porous in $(\mathscr{A}, d)$.

Lemma 4.3. Let $m \in \mathbb{N}$ and suppose $f \in E_{m}$. Let $x_{f} \in C_{\infty}$ with $f\left(x_{f}\right)=\inf \left(f ; C_{\infty}\right)$. If

$$
f_{\gamma}(x)=f(x)+\gamma\left\|x-x_{f}\right\|
$$

is defined for each $\gamma>0$ and all $x \in C_{1}$, then $f_{\gamma} \in \mathscr{A}$ and $d\left(f_{\gamma}, f\right) \leq \gamma$.

Proof of Lemma 4.3. Clearly $f_{\gamma} \in \mathcal{A}$. We will estimate $d\left(f_{\gamma}, f\right)$. Since

$$
\varphi\left(x_{f}\right) \leq f\left(x_{f}\right)=\inf \left(f ; C_{\infty}\right)
$$

it follows from property $\left(Q_{2}\right)$ that $\left\|x_{f}\right\| \leq m$. For each $k=1,2, \ldots$ we have

$$
\begin{aligned}
d_{k}\left(f_{\gamma}, f\right) & =\sup \left\{\left|f_{\gamma}(x)-f(x)\right| \mid x \in C_{1} \text { and }\|x\| \leq k\right\} \\
& =\sup \left\{\gamma\left\|x-x_{f}\right\| \mid x \in C_{1} \text { and }\|x\| \leq k\right\} \\
& \leq \gamma \sup \left\{\|x\|+\left\|x_{f}\right\| \mid x \in C_{1} \text { and }\|x\| \leq k\right\} \\
& \leq \gamma(m+k)
\end{aligned}
$$

and hence

$$
d\left(f_{\gamma}, f\right) \leq \sum_{k=1}^{\infty} 2^{-k} \gamma(m+k)(\gamma(m+k)+1)^{-1} \leq \gamma \sum_{k=1}^{\infty} 2^{-k}=\gamma
$$

Lemma 4.4. Let $m, n \in \mathbb{N}$. Choose a real number $r$ with $0<r \leq 1$ and choose real numbers $\gamma=\gamma(r)=\left(1-1 / 2^{m+3}\right) r$ and $\theta=\theta(r, m, n)=r /\left(2^{m+4} n\right)$. If $f \in E_{m}$ and $g \in \mathcal{A}$ with $d\left(g, f_{\gamma}\right) \leq \theta$, then $g \in \mathscr{F}_{n}$ and $d(g, f)<r$.

Proof of Lemma 4.4. Let $f \in E_{m}, g \in \mathscr{A}$, and $d\left(g, f_{\gamma}\right) \leq \theta$. Then by elementary algebra we can show that $d_{m}\left(g, f_{\gamma}\right) \leq 2^{m+1} \theta$. If $x \in C_{i}$ and $\varphi(x) \leq \inf \left(f ; C_{i}\right)+1$, then $\varphi(x) \leq$ $\inf \left(f ; C_{\infty}\right)+1$ and since $f \in E_{m}$ it follows that $\|x\| \leq m$. Hence

$$
\left|g(x)-f_{\gamma}(x)\right| \leq 2^{m+1} \theta .
$$


Note also that if $y \in C_{i}$ and $f_{\gamma}(y) \leq \inf \left(f_{\gamma} ; C_{i}\right)+1$, then $\varphi(y) \leq f_{\gamma}(y) \leq \inf \left(f_{\gamma} ; C_{i}\right)+$ $1 \leq \inf \left(f_{\gamma} ; C_{\infty}\right)+1$ and $\operatorname{since} \inf \left(f_{\gamma} ; C_{\infty}\right)=f_{\gamma}\left(x_{f}\right)=f\left(x_{f}\right)=\inf \left(f ; C_{\infty}\right)$ it follows that $\varphi(y) \leq \inf \left(f ; C_{\infty}\right)+1$. Since $f \in E_{m}$ we deduce that $\|y\| \leq m$ and hence also that

$$
\left|g(y)-f_{\gamma}(y)\right| \leq 2^{m+1} \theta \text {. }
$$

Since this holds for any such $y$, we obtain

$$
\inf \left(g ; C_{i}\right) \leq \inf \left(f_{\gamma} ; C_{i}\right)+2^{m+1} \theta .
$$

Assume that $z \in C_{i}$ and $g(z) \leq \inf \left(g ; C_{i}\right)+2^{m+1} \theta$ then $\varphi(z) \leq g(z) \leq \inf \left(g ; C_{i}\right)+$ $2^{m+1} \theta \leq \inf \left(f_{\gamma} ; C_{i}\right)+2^{m+2} \theta<\inf \left(f_{\gamma} ; C_{\infty}\right)+1=\inf \left(f ; C_{\infty}\right)+1$. Hence $\|z\| \leq m$ and

$$
\left|g(z)-f_{\gamma}(z)\right| \leq 2^{m+1} \theta \text {. }
$$

We can now deduce that $f_{\gamma}(z) \leq g(z)+2^{m+1} \theta \leq \inf \left(g ; C_{i}\right)+2^{m+2} \theta \leq \inf \left(f_{\gamma} ; C_{i}\right)+3.2^{m+1} \theta$ $\leq \inf \left(f, C_{\infty}\right)+3.2^{m+1} \theta$. If we choose $p$ so large that $\inf \left(f ; C_{i}\right) \geq \inf \left(f, C_{\infty}\right)-2^{m+1} \theta$ when $i \in\{p, p+1, \ldots\} \cup\{\infty\}$, then

$$
f_{\gamma}(z) \leq \inf \left(f, C_{i}\right)+2^{m+3} \theta \leq f(z)+2^{m+3} \theta
$$

and hence

$$
\left\|z-x_{f}\right\| \leq 2^{m+3} \frac{\theta}{\gamma} \leq \frac{1}{n}
$$

Since $z \in C_{i}$ and $g(z) \leq \inf \left(g ; C_{i}\right)+2^{m+1} \theta$ for $i \in\{p, p+1, \ldots\} \cup\{\infty\}$ implies that $\| z-$ $x_{f} \| \leq 1 / n$, we have shown that $g \in \mathscr{F}_{n}$. Finally we note that $d(g, f) \leq d\left(g, f_{\gamma}\right)+d\left(f_{\gamma}, f\right) \leq$ $\theta+\gamma<r$.

We can now complete the proof of the main result.

Proof of Theorem 2.1. In Remark 4.2, we observed that the proof of Theorem 2.1 would be complete if we could show that for each $m, n \in \mathbb{N}$ the set $E_{m} \backslash \mathscr{F}_{n}$ is porous in $(\mathscr{A}, d)$. Let $f \in E_{m} \backslash \mathscr{F}_{n}$. Choose any real number $r$ with $0<r \leq 1$ and choose real numbers $\gamma=\gamma(r)$ and $\theta=\theta(r, m, n)$ as in Lemma 4.4. If we define $\alpha=\alpha(m, n)$ by the formula

$$
\alpha=\frac{1}{\left(2^{m+4} n\right)}
$$

then $\theta=\alpha r$ and for each $r$ with $0<r \leq 1$ we can see from Lemma 4.4 that

$$
\left\{g \in \mathscr{A} \mid d\left(g, f_{\gamma}\right) \leq \alpha r\right\} \subset\{g \in \mathscr{A} \mid d(g, f) \leq r\} \cap \mathscr{F}_{n} .
$$

Hence each sufficiently small ball $B_{d}(f, r) \subset(\mathscr{A}, d)$ centred at a point $f \in E_{m} \backslash \mathscr{F}_{n}$ contains a smaller ball $B_{d}\left(f_{\gamma}, \alpha r\right)$ of fixed proportional radius centred at the point $f_{\gamma}$ and lying entirely within $\mathscr{F}_{n}$. Hence $E_{m} \backslash \mathscr{F}_{n}$ is porous in $(\mathscr{A}, d)$. This completes the proof. 


\section{References}

[1] E. Asplund, Fréchet differentiability of convex functions, Acta Math. 121 (1968), 31-47.

[2] J. M. Ball and N. S. Nadirashvili, Universal singular sets for the one-dimensional variational problems, Calc. Var. Partial Differential Equations 1 (1993), no. 4, 429-438.

[3] G. Beer and R. Lucchetti, Convex optimization and the epi-distance topology, Trans. Amer. Math. Soc. 327 (1991), no. 2, 795-813.

[4] A. Cellina and C. Mariconda, The existence question in the calculus of variations: a density result, Proc. Amer. Math. Soc. 120 (1994), no. 4, 1145-1150.

[5] F. S. De Blasi and J. Myjak, Sur la porosité de l'ensemble des contractions sans point fixe, C. R. Acad. Sci. Paris Sér. I Math. 308 (1989), no. 2, 51-54 (French).

[6] _ _ On a generalized best approximation problem, J. Approx. Theory 94 (1998), no. 1, 5472.

[7] R. Deville and J. P. Revalski, Porosity of ill-posed problems, Proc. Amer. Math. Soc. 128 (2000), no. $4,1117-1124$.

[8] P. G. Howlett and A. J. Zaslavski, On the minimization of convex functions in reflexive Banach spaces, Commun. Appl. Anal. 5 (2001), no. 4, 535-545.

[9] A. D. Ioffe and A. J. Zaslavski, Variational principles and well-posedness in optimization and calculus of variations, SIAM J. Control Optim. 38 (2000), no. 2, 566-581.

[10] M. M. Israel Jr. and S. Reich, Extension and selection problems for nonlinear semigroups in Banach spaces, Math. Japon. 28 (1983), no. 1, 1-8.

[11] S. Reich and A. J. Zaslavski, Generic convergence of descent methods in Banach spaces, Math. Oper. Res. 25 (2000), no. 2, 231-242.

[12] - The set of divergent descent methods in a Banach space is $\sigma$-porous, SIAM J. Optim. 11 (2001), no. 4, 1003-1018.

[13] - The set of noncontractive mappings is $\sigma$-porous in the space of all nonexpansive mappings, C. R. Acad. Sci. Paris Sér. I Math. 333 (2001), no. 6, 539-544.

[14] J. Semple, Infinite positive-definite quadratic programming in a Hilbert space, J. Optim. Theory Appl. 88 (1996), no. 3, 743-749.

[15] G. Vidossich, Most of the successive approximations do converge, J. Math. Anal. Appl. 45 (1974), $127-131$.

[16] L. Zajíček, Porosity and $\sigma$-porosity, Real Anal. Exchange 13 (1987/1988), no. 2, 314-350.

[17] Small non- $\sigma$-porous sets in topologically complete metric spaces, Colloq. Math. 77 (1998), no. 2, 293-304.

[18] A. J. Zaslavski, Dynamic properties of optimal solutions of variational problems, Nonlinear Anal. 27 (1996), no. 8, 895-931.

[19] Generic well-posedness of optimal control problems without convexity assumptions, SIAM J. Control Optim. 39 (2000), no. 1, 250-280.

[20] - Existence of solutions of optimal control problems for a generic integrand without convexity assumptions, Nonlinear Anal. Ser. A: Theory Methods 43 (2001), no. 3, 339-361.

[21] Well-posedness and porosity in optimal control without convexity assumptions, Calc. Var. Partial Differential Equations 13 (2001), no. 3, 265-293.

P. G. Howlett: Centre for Industrial and Applied Mathematics (CIAM), University of South Australia, Mawson Lakes, SA 5059, Australia

E-mail address: phil.howlett@unisa.edu.au

A. J. Zaslavski: Department of Mathematics, Technion - Israel Technology Institute, Haifa 32000, Israel

E-mail address: ajzasl@tx.technion.ac.il 


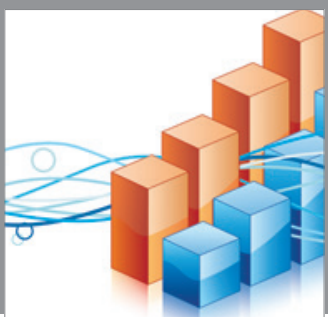

Advances in

Operations Research

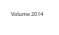

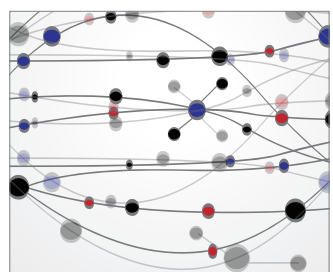

\section{The Scientific} World Journal
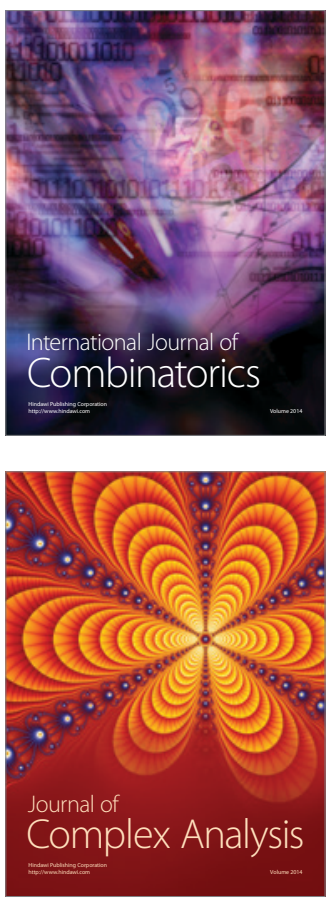

International Journal of

Mathematics and

Mathematical

Sciences
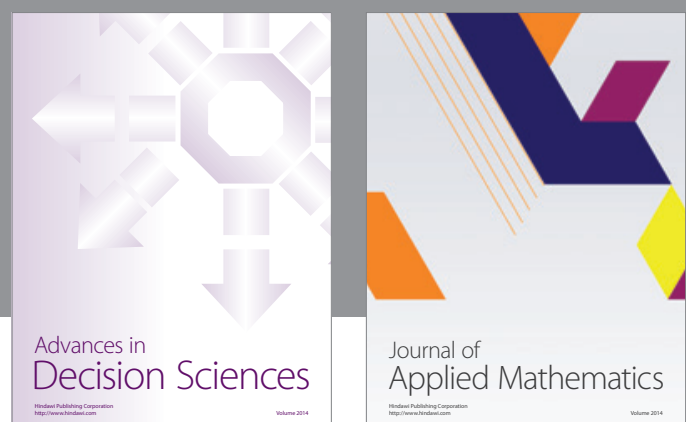

Journal of

Applied Mathematics
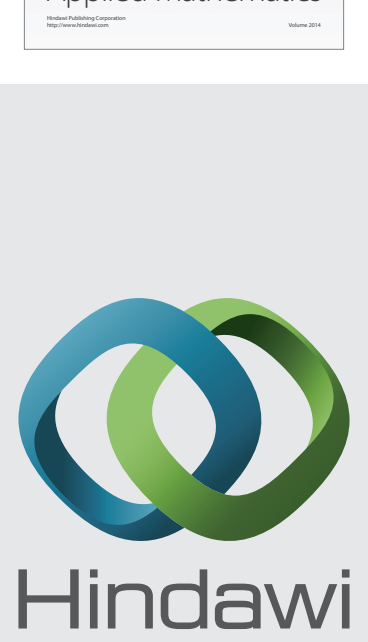

Submit your manuscripts at http://www.hindawi.com
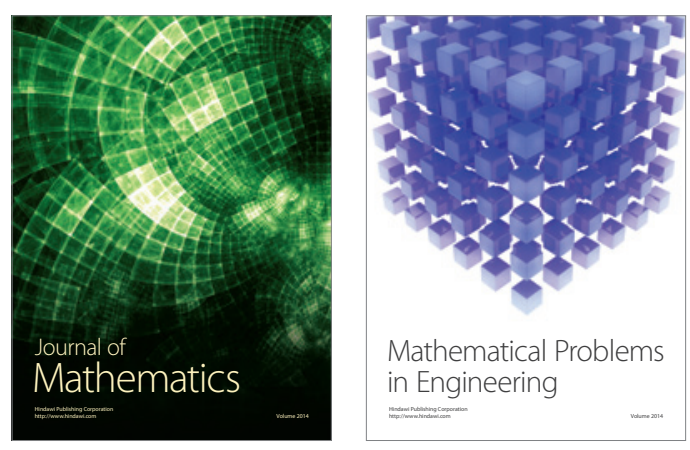

Mathematical Problems in Engineering
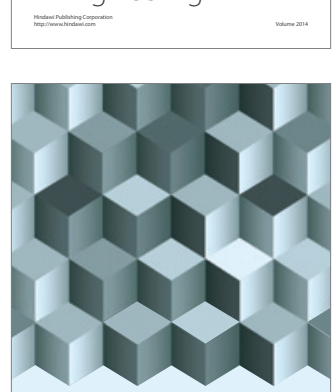

Journal of

Function Spaces
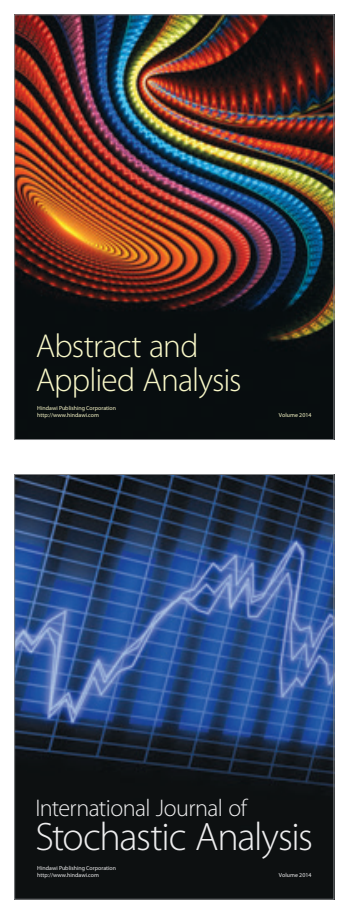

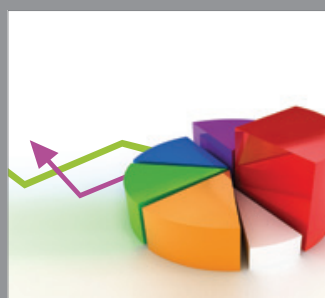

ournal of

Probability and Statistics

Promensencen
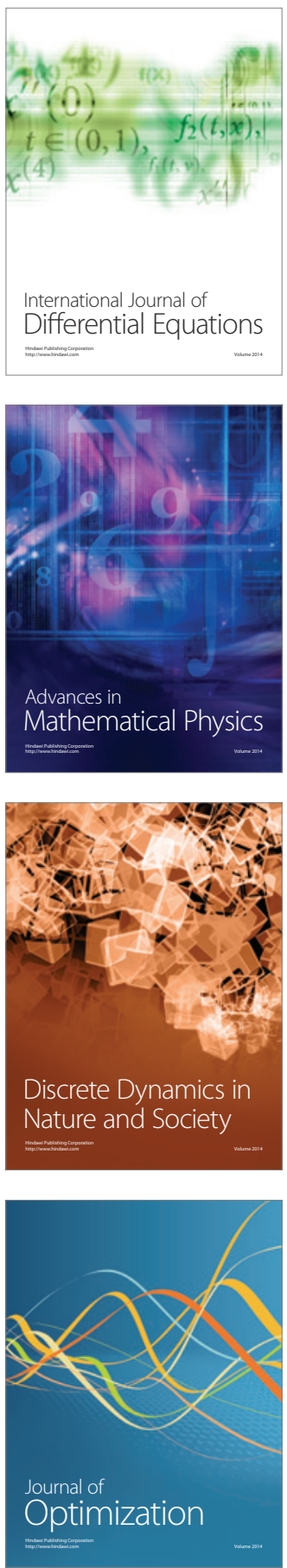